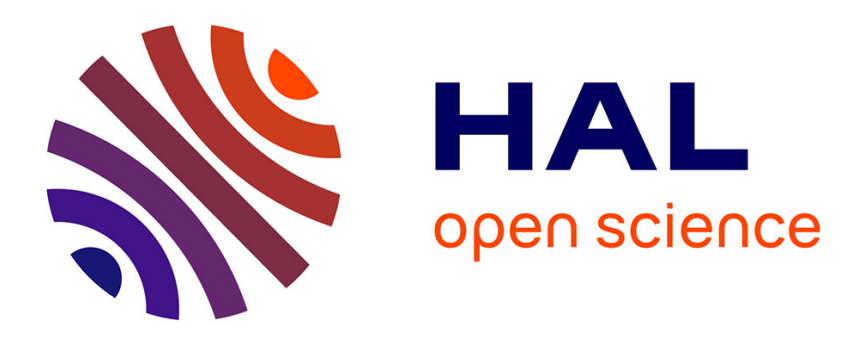

\title{
Asymptotic heat equation for crossing superconductive thin walls
}

Isabelle Gruais, Dan Polisevski

\section{To cite this version:}

Isabelle Gruais, Dan Polisevski. Asymptotic heat equation for crossing superconductive thin walls. Applicable Analysis, 2012, 91 (11), pp.2029-2043. hal-00487728

\section{HAL Id: hal-00487728 \\ https://hal.science/hal-00487728}

Submitted on 30 May 2012

HAL is a multi-disciplinary open access archive for the deposit and dissemination of scientific research documents, whether they are published or not. The documents may come from teaching and research institutions in France or abroad, or from public or private research centers.
L'archive ouverte pluridisciplinaire HAL, est destinée au dépôt et à la diffusion de documents scientifiques de niveau recherche, publiés ou non, émanant des établissements d'enseignement et de recherche français ou étrangers, des laboratoires publics ou privés. 


\title{
ASYMPTOTIC HEAT EQUATION FOR CROSSING SUPERCONDUCTIVE THIN WALLS
}

\author{
ISABELLE GRUAIS * AND DAN POLIŠEVSKI **
}

\begin{abstract}
This work deals with the homogenization of the nonstationary heat conduction which takes place in a binary three-dimensional medium consisting of an ambiental phase having conductivity of unity order and a set of highly conductive thin walls crossing orthogonally and periodically. This situation covers in fact three types of microstructures, usually called: box-type (or honeycomb), gridwork and layered. The study is based on the energetic procedure of homogenization associated to a control-zone method, specific to the geometry of the microstructure and to the singularity of the conductivity coefficients. In the present case the main result is the system that governs the asymptotic behaviour of the temperature distribution in this binary medium. It displays a significant increase of the conductivity due to the superconductive thin walls, revealing their seemingly paradoxal behaviour of having an everlasting action on the environment, in spite of an obvious vanishing volume. Moreover, the dependence of this behaviour with respect to the relative thicknesses of the walls can be detailed.
\end{abstract}

Mathematical Subject Classification (2000). 35B27, 35K57, 76R50.

Keywords. homogenization, conduction, fine-scale, box-type structure.

\section{INTRODUCTION}

The foundations of homogenized layered materials were laid down by Murat and Tartar in their pioneering work [11]. This method was still used by [10] in the framework of weaker topologies. The case of $B V$-functions and sequences of measures is worked out in [6]. Many examples and applications may be found in [1]. The difficulties arising from the direct computation of the behaviour of these structures are twofold: the great number of cells and the small thickness of the material. The periodic distribution classically suggests that the homogenization method for perforated domains should be used as in [12], where the period $\varepsilon$ and the thickness $\delta$ of the material are considered as independent vanishing parameters. A classical issue is then to consider the problem of permuting both converging processes. This is made in details in [12]. As the practical point of view favors geometric consideration, measures provide a more general tool when they are confined to the description of the critical part of a system, as in [2] and [3]. This approach was used in [13], where the homogenization of elastic reticulated structures is performed for an anisotropic material surrounded by an empty environment.

Our study is based on the control-zone method which was introduced and developed by [4]-[5] and [7]-[8], specific to the binary composition of the system, namely the fine substructure and the ambiental phase. This procedure proved its efficiency 
in the modelling of fine substructures where concentrated material of high contribution influences the behavior of the macroscopic problem in spite of its vanishing volume. We are here in a case when the fine substructure does not have a macroscopic capacity. In the case when such a capacity exists, the coupling between the fine substructure and the ambient is made through a so-called rarefying ratio $\gamma_{\varepsilon}$, which arises as a criterium for the existence of the two-temperature model. Here, unlike the critical case studied in [5], the connections between the layers of the reticulated structure annihilate the capacitary term $\lim _{\varepsilon \rightarrow 0} \gamma_{\varepsilon}$ when it is defined. This was also observed in [8] in the absence of connections. As the rarefaction number is in fact zero, we obtain here a one-temperature macroscopic model. Nevertheless, the consideration of a transmission problem between the two components of our medium modifies the asymptotic behaviour in a way that cannot be anticipated from [12]-[13], because the sequence of problems under consideration behaves singularly with respect to the period of the structure.

The present paper is organized as follows:

Section 2 is devoted to the main notations and to the study of the governing problem. We set the functional framework for which the existence and uniqueness of the solution can be established.

In Section 3 we present two operators that have a localizing effect and that we use in order to obtain the specific inequalities related to the box-type structure. Considering natural control zones, we define the specific caracterizing functions and the step approximation operators which are associated to it.

Section 4 deals with the homogenization process, overcoming the singular behavior of the energy term when $\varepsilon$ tends to zero. We obtain the homogenized equation which presents an explicit formula for the effective conductivity coefficients. It displays a significant increase of the conductivity due to the superconductive thin walls, revealing their seemingly paradoxal behaviour of having an everlasting action on the environment, in spite of an obvious vanishing volume. Moreover, the dependence of this behaviour with respect to the relative thicknesses of the walls can be detailed. The proof relies on the energetic method adapted to our control-zone context. No critical thickness of the layers appears and the intersections of the layers have no distinct influence upon the asymptotic behaviour of the temperature.

\section{Setting of the PRoblem}

Let $L>0, T>0, \Omega=]-\frac{L}{2}, \frac{L}{2}[3=]-\frac{1}{2}, \frac{1}{2}[$ and $n \in \mathrm{N}$. From now on we use the notations

$$
\varepsilon=\frac{L}{2 n+1}, \quad \mathbf{Z}_{\varepsilon}=\{k \in \mathbf{Z}, \quad|k| \leq n\} \quad \text { and } \quad I_{\varepsilon}^{k}=\varepsilon k+\varepsilon I, \quad k \in \mathbf{Z}_{\varepsilon} .
$$

Obviously, $\operatorname{card} \mathbf{Z}_{\varepsilon}=L / \varepsilon$ and $\left.x \in\right]-\frac{L}{2}, \frac{L}{2}\left[\right.$ if and only if there exists $k \in \mathbf{Z}_{\varepsilon}$ such that $x \in \bar{I}_{\varepsilon}^{k}$.

For any $i \in\{1,2,3\}$, we consider $r_{i}^{\varepsilon} \geq 0, r_{i}^{\varepsilon}<<\varepsilon$, that is $\frac{r_{i}^{\varepsilon}}{\varepsilon} \rightarrow 0$ when $\varepsilon \rightarrow 0$. For any $k \in \mathbf{Z}_{\varepsilon}$ and $i \in\{1,2,3\}$, we define:

$$
\begin{gathered}
D_{\varepsilon, k}^{i}=\left\{x=\left(x_{i}, x_{2}, x_{3}\right) \in \Omega,\left|x_{i}-\varepsilon k\right|<r_{i}^{\varepsilon}\right\}, \\
D_{\varepsilon}=\cup_{i=1}^{3} D_{\varepsilon}^{i}, \quad D_{\varepsilon}^{i}=\cup_{k \in \mathbf{Z}_{\varepsilon}} D_{\varepsilon, k}^{i} .
\end{gathered}
$$


We consider that $\Omega$ is occupied by two materials with highly different conductivities; one is highly conductive and it is concentrated in the vanishing domain $D_{\varepsilon}$ (notice that $\left|D_{\varepsilon}^{i}\right|=O\left(r_{i}^{\varepsilon} / \varepsilon\right)$ ) and the other lies in $\Omega_{\varepsilon}:=\Omega \backslash D_{\varepsilon}$ and has conductivity of unity order.

We study the asymptotic behaviour with respect to $\varepsilon(\varepsilon \rightarrow 0)$ of the nonstationary heat conduction process in $\Omega$. Assuming that we know the densities and the conductivities of the two materials, the source term and the initial distribution of the temperature, the problem that the temperature $u^{\varepsilon}$ satisfies in some sense is the following:

To find $u^{\varepsilon}$ solution of

$$
\begin{gathered}
\rho^{\varepsilon} \frac{\partial u^{\varepsilon}}{\partial t}-\operatorname{div}\left(k^{\varepsilon} \nabla u^{\varepsilon}\right)=f^{\varepsilon} \quad \text { in } \Omega^{T} \\
{\left[u^{\varepsilon}\right]_{\varepsilon}=0 \quad \text { on } \quad\left(\partial D_{\varepsilon} \backslash \partial \Omega\right)^{T}} \\
{\left[k^{\varepsilon} \nabla u^{\varepsilon}\right]_{\varepsilon} n=0 \quad \text { on } \quad\left(\partial D_{\varepsilon} \backslash \partial \Omega\right)^{T}} \\
u^{\varepsilon}=0 \quad \text { on } \quad(\partial \Omega)^{T} \\
u^{\varepsilon}(0)=u_{0}^{\varepsilon} \quad \text { in } \Omega
\end{gathered}
$$

where $[\cdot]_{\varepsilon}$ is the jump across the interface $\partial D_{\varepsilon}, n$ is the normal on $\partial D_{\varepsilon}$ in the outward direction,

$$
\begin{aligned}
& f^{\varepsilon} \rightarrow f \quad \text { weakly in } \quad L^{2}\left(0, T ; H^{-1}(\Omega)\right) \\
& u_{0}^{\varepsilon} \rightarrow u_{0} \quad \text { weakly in } \quad L^{2}(\Omega) \\
& \rho^{\varepsilon}(x)=\left\{\begin{array}{llll}
\rho_{a} & \text { if } & x \in \Omega_{\varepsilon} & \left(\rho_{a}>0\right) \\
\rho_{b} & \text { if } & x \in D_{\varepsilon} & \left(\rho_{b}>0\right)
\end{array}\right. \\
& k_{i j}^{\varepsilon}(x)= \begin{cases}a_{i j}(x) & \text { if } \quad x \in \Omega_{\varepsilon} \\
\frac{1}{\left|D_{\varepsilon}\right|} \sum_{k=1}^{3} b_{k}(x) \delta_{i k} \delta_{j k} & \text { if } \quad x \in D_{\varepsilon},\end{cases}
\end{aligned}
$$

where $b_{k} \in C^{2}(\bar{\Omega}), b_{k}(x)>0, \forall x \in \bar{\Omega}$, and $a_{i j} \in L^{\infty}(\Omega)$ with the property that there exists $\alpha>0$ such that

$$
a_{i j}(x) \xi_{i} \xi_{j} \geq \alpha|\xi|^{2}, \quad \forall \xi \in \mathbf{R}^{N} \text { and for a.a. } \quad x \in \Omega .
$$

Throughout this paper, we use the following compact notation for the cylindrical time-domain, as we already did in (4)-(7):

$$
\left.E^{T}:=E \times\right] 0, T[, \quad \forall E \subseteq \Omega .
$$

Let $H_{\varepsilon}$ be the Hilbert space $L^{2}(\Omega)$ endowed with the scalar product

$$
(u, v)_{H_{\varepsilon}}:=\left(\rho^{\varepsilon} u, v\right)_{\Omega} .
$$

As $H_{0}^{1}(\Omega)$ is dense in $H_{\varepsilon}$ for any fixed $\varepsilon>0$, we can set

$$
H_{0}^{1}(\Omega) \subseteq H_{\varepsilon} \simeq H_{\varepsilon}^{\prime} \subseteq H^{-1}(\Omega)
$$

with continuous embeddings.

Now, we can present the variational formulation of the problem (4)-(8). 
To find $u^{\varepsilon} \in L^{2}\left(0, T ; H_{0}^{1}(\Omega)\right) \cap L^{\infty}\left(0, T ; H_{\varepsilon}\right)$ satisfying (in some sense) the initial condition (8) and the following equation

$$
\frac{d}{d s}\left(u^{\varepsilon}, w\right)_{H_{\varepsilon}}+\left(k_{\varepsilon} \nabla u^{\varepsilon}, \nabla w\right)_{\Omega}=\left\langle f^{\varepsilon}, w\right\rangle \quad \text { in } \quad \mathcal{D}^{\prime}(0, T), \quad \forall w \in H_{0}^{1}(\Omega)
$$

where $\langle\cdot, \cdot\rangle$ denotes the duality product between $H^{-1}(\Omega)$ and $H_{0}^{1}(\Omega)$.

Theorem 2.1. Under the above hypotheses and notations, problem (17) has a unique solution. Moreover, $\frac{d u^{\varepsilon}}{d s} \in L^{2}\left(0, T ; H^{-1}(\Omega)\right)$ and hence, $u^{\varepsilon}$ is equal almost everywhere to a function of $C^{0}\left([0, T] ; H_{\varepsilon}\right)$; this is the sense of the initial condition (8).

From now on, for any $E \subset \Omega$, we use the notations

$$
|\cdot|_{E}:=|\cdot|_{L^{2}(E)} \quad \text { and } \quad f_{E} \cdot d x:=\frac{1}{|E|} \int_{E} \cdot d x
$$

Proposition 2.2. Recalling the definition (1) of $\varepsilon$, we have

$$
\left(u^{\varepsilon}\right)_{\varepsilon>0} \quad \text { is bounded in } L^{\infty}\left(0, T ; L^{2}(\Omega)\right) \cap L^{2}\left(0, T ; H_{0}^{1}(\Omega)\right) \text {. }
$$

Moreover, there exists $C>0$, independent of $\varepsilon$, such that

$$
\int_{0}^{T} f_{D_{\varepsilon}}\left|\nabla u^{\varepsilon}\right|^{2} \leq C
$$

Proof. If we substitute $w=u^{\varepsilon}$ in the variational problem (17), integrate over $(0, t)$ for any $t \in] 0, T$, use (13) and denote $\beta=\min \left\{\min _{\bar{\Omega}} b_{k}, 1 \leq k \leq 3\right\}$, as $\beta>0$, then we get:

$$
\begin{gathered}
\frac{1}{2}\left(\rho_{a}\left|u^{\varepsilon}(t)\right|_{\Omega_{\varepsilon}}^{2}+\rho_{b}\left|u^{\varepsilon}(t)\right|_{D_{\varepsilon}}^{2}\right)+\alpha \int_{0}^{t}\left|\nabla u^{\varepsilon}\right|_{\Omega_{\varepsilon}}^{2} d s+\beta \int_{0}^{t} f_{D_{\varepsilon}}\left|\nabla u^{\varepsilon}\right|^{2} d s \leq \\
\leq \int_{0}^{t}\left\langle f^{\varepsilon}(s), u^{\varepsilon}(s)\right\rangle d s+\frac{1}{2}\left(\rho_{a}\left|u_{0}^{\varepsilon}\right|_{\Omega_{\varepsilon}}^{2}+\rho_{b}\left|u_{0}^{\varepsilon}\right|_{D_{\varepsilon}}^{2}\right) .
\end{gathered}
$$

Using the boundedness properties that follow from (9) and (10), we obtain easily:

$$
\left|u^{\varepsilon}(t)\right|_{\Omega}^{2}+\int_{0}^{t}\left|\nabla u^{\varepsilon}\right|_{\Omega}^{2} d s \leq C,
$$

which proves (19). The proof is completed by recalling (21).

\section{The CONTROL-ZONE TOOLS}

Definition 3.1. For any $u \in L^{2}\left(0, T ; H^{1}(\Omega)\right), i \in\{1,2,3\}$ and $k \in \mathbf{Z}_{\varepsilon}$ we define $G_{\varepsilon, k}^{i}(u) \in L^{2}\left(0, T ; H^{1 / 2}\left(\Omega^{\prime}\right)\right)$ and $G_{\varepsilon}^{i}(u) \in L^{2}\left(\Omega^{T}\right)$ by

$$
\begin{gathered}
G_{\varepsilon, k}^{i}(u)\left(t, x_{i}^{\prime}\right)=\frac{1}{2}\left(u\left(t, \varepsilon k-r_{i}^{\varepsilon}, x_{i}^{\prime}\right)+u\left(t, \varepsilon k+r_{i}^{\varepsilon}, x_{i}^{\prime}\right)\right), \\
G_{\varepsilon}^{i}(u)\left(t, x_{i}, x_{i}^{\prime}\right)=\sum_{k \in \mathbf{Z}_{\varepsilon}} G_{\varepsilon, k}^{i}(u)\left(t, x_{i}^{\prime}\right) 1_{I_{\varepsilon}^{k}}\left(x_{i}\right),
\end{gathered}
$$

where $\left.t \in[0, T], x_{i} \in\right]-\frac{L}{2}, \frac{L}{2}\left[\right.$ and $\left.x_{i}^{\prime}:=\left(\cdots, \not x_{i}, \cdots\right) \in \Omega^{\prime}:=\right]-\frac{L}{2}, \frac{L}{2}\left[^{2}\right.$. 
Proposition 3.2. For any $u \in L^{2}\left(0, T ; H^{1}(\Omega)\right)$ and $i \in\{1,2,3\}$, we have:

$$
\int_{0}^{T} f_{D_{\varepsilon}^{i}}\left|G_{\varepsilon}^{i}(u)\right|^{2}=f_{\Omega}\left|G_{\varepsilon}^{i}(u)\right|^{2} .
$$

Proof. For almost all $t \in] 0, T[$, we have

$$
\int_{\Omega}\left|G_{\varepsilon}^{i}(u)\right|^{2}=\sum_{k \in \mathbf{Z}_{\varepsilon}} \int_{I \times \Omega^{\prime}}\left|G_{r_{i}^{\varepsilon}, k}^{i}(u)\left(s, x_{i}^{\prime}\right)\right|^{2} 1_{I_{\varepsilon}^{k}}=\varepsilon \sum_{k \in \mathbf{Z}_{\varepsilon}} \int_{\Omega^{\prime}}\left|G_{r_{i}^{\varepsilon}, k}^{i}(u)\right|^{2} .
$$

Moreover:

$$
\begin{gathered}
\int_{D_{\varepsilon}^{i}}\left|G_{\varepsilon}^{i}(u)\right|^{2}=\frac{1}{2 \operatorname{card} \mathbf{Z}_{\varepsilon} r_{i}^{\varepsilon}\left|\Omega^{\prime}\right|} \sum_{k \in \mathbf{Z}_{\varepsilon}} \int_{I_{r_{\varepsilon}}^{k} \times \Omega^{\prime}}\left|G_{r_{i}^{\varepsilon}, k}^{i}(u)\right|^{2}\left(t, x_{i}^{\prime}\right) 1_{I_{\varepsilon}^{k}}\left(x_{i}\right)= \\
=\frac{\varepsilon}{L\left|\Omega^{\prime}\right|} \sum_{k \in \mathbf{Z}_{\varepsilon}} \int_{\Omega^{\prime}}\left|G_{r_{i}^{\varepsilon}, k}^{i}(u)\right|^{2} .
\end{gathered}
$$

This achieves the proof.

Proposition 3.3. There exists $C>0$ independent of $\varepsilon$ such that, for any $u \in$ $L^{2}\left(0, T ; H^{1}(\Omega)\right), i \in\{1,2,3\}$ and $k \in \mathbf{Z}_{\varepsilon}$, there holds:

$$
\begin{aligned}
\left|u-G_{\varepsilon, k}^{i}(u)\right|_{L^{2}\left(0, T ; I_{\varepsilon}^{k} \times \Omega^{\prime}\right)} & \leq C \varepsilon\left|\frac{\partial u}{\partial x_{i}}\right|_{L^{2}\left(0, T ; I_{\varepsilon}^{k} \times \Omega^{\prime}\right)} \\
\left|u-G_{\varepsilon, k}^{i}(u)\right|_{L^{2}\left(0, T ; I_{r_{i}^{\varepsilon}}^{k} \times \Omega^{\prime}\right)} & \leq C r_{i}^{\varepsilon}\left|\frac{\partial u}{\partial x_{i}}\right|_{L^{2}\left(0, T ; I_{r_{i}}^{k} \times \Omega^{\prime}\right)}
\end{aligned}
$$

where $x=\left(x_{i}, x_{i}^{\prime}\right) \in I_{\varepsilon}^{k} \times \Omega^{\prime}$ and $I_{r_{i}^{\varepsilon}}^{k}=\varepsilon k+2 r_{i}^{\varepsilon} I$.

Proof. Obviously, it is sufficient to prove that we have similar uniform inequalities in $H^{1}(\Omega)$. First, for any $u \in H^{1}(\Omega)$, we consider the following quantity:

As we have

$$
J_{\varepsilon}^{k}=\int_{I_{\varepsilon}^{k} \times \Omega^{\prime}}\left|u-G_{\varepsilon, k}^{i}(u)\right|^{2} .
$$

$$
\begin{gathered}
J_{\varepsilon}^{k}=\int_{I_{\varepsilon}^{k}} d x_{i} \int_{\Omega^{\prime}}\left|u\left(x_{i}, x_{i}^{\prime}\right)-\frac{1}{2}\left(u\left(\varepsilon k-r_{i}^{\varepsilon}, x_{i}^{\prime}\right)+u\left(\varepsilon k+r_{i}^{\varepsilon}, x_{i}^{\prime}\right)\right)\right|^{2} d x_{i}^{\prime}= \\
=\frac{1}{4} \int_{I_{\varepsilon}^{k}} d x_{i} \int_{\Omega^{\prime}}\left|\int_{\varepsilon k-r_{i}^{\varepsilon}}^{x_{i}} \frac{\partial u}{\partial x_{i}}\left(s, x_{i}^{\prime}\right) d s-\int_{x_{i}}^{\varepsilon k+r_{i}^{\varepsilon}} \frac{\partial u}{\partial x_{i}}\left(s, x_{i}^{\prime}\right) d s\right|^{2} d x_{i}^{\prime} \leq \\
\leq \frac{1}{2} \int_{I_{\varepsilon}^{k}} d x_{i} \int_{\Omega^{\prime}}\left(\left|\int_{\varepsilon k-r_{i}^{\varepsilon}}^{x_{i}} \frac{\partial u}{\partial x_{i}}\left(s, x_{i}^{\prime}\right) d s\right|^{2}+\left|\int_{x_{i}}^{\varepsilon k+r_{i}^{\varepsilon}} \frac{\partial u}{\partial x_{i}}\left(s, x_{i}^{\prime}\right) d s\right|^{2}\right) d x_{i}^{\prime} \leq \\
\leq \frac{1}{2} \int_{I_{\varepsilon}^{k}} d x_{i} \int_{\Omega^{\prime}}\left[\left(x_{i}-\varepsilon k+r_{i}^{\varepsilon}\right) \int_{\varepsilon k-r_{i}^{\varepsilon}}^{x_{i}}\left|\frac{\partial u}{\partial x_{i}}\left(s, x_{i}^{\prime}\right)\right|^{2} d s+\left(\varepsilon k+r_{i}^{\varepsilon}-x_{i}\right) \int_{x_{i}}^{\varepsilon k+r_{i}^{\varepsilon}}\left|\frac{\partial u}{\partial x_{i}}\left(s, x_{i}^{\prime}\right)\right|^{2} d s\right] d x_{i}^{\prime},
\end{gathered}
$$

there results

$$
J_{\varepsilon}^{k} \leq \frac{\varepsilon}{2} \int_{I_{\varepsilon}^{k}} d x_{i} \underbrace{\int_{\Omega^{\prime}} d x_{i}^{\prime} \int_{\varepsilon k-\frac{\varepsilon}{2}}^{\varepsilon k+\frac{\varepsilon}{2}}\left|\frac{\partial u}{\partial x_{i}}\left(s, x_{i}^{\prime}\right)\right|^{2} d s}_{\text {independent of } x_{i}}=\frac{\varepsilon^{2}}{2} \int_{I_{\varepsilon}^{k} \times \Omega^{\prime}}\left|\frac{\partial u}{\partial x_{i}}\right|^{2},
$$

from which (25) follows. 
Second, still for $u \in H^{1}(\Omega)$, we have

$$
\begin{aligned}
& \int_{I_{r_{i}^{\varepsilon}}^{k} \times \Omega^{\prime}}\left|u-G_{\varepsilon, k}^{i}(u)\right|^{2}=\int_{I_{r_{i}^{\varepsilon}}^{k}} d x_{i} \int_{\Omega^{\prime}}\left|u\left(x_{i}, x_{i}^{\prime}\right)-\frac{1}{2}\left(u\left(\varepsilon k-r_{i}^{\varepsilon}, x_{i}^{\prime}\right)+u\left(\varepsilon k+r_{i}^{\varepsilon}, x_{i}^{\prime}\right)\right)\right|^{2} d x_{i}^{\prime} d x_{i} \leq \\
& \leq \frac{1}{2} \int_{I_{r_{i}^{\varepsilon}}^{k}} \int_{\Omega^{\prime}}\left(\left|\int_{\varepsilon k-r_{i}^{\varepsilon}}^{x_{i}} \frac{\partial u}{\partial x_{i}}\left(s, x_{i}^{\prime}\right) d s\right|^{2}+\left|\int_{x_{i}}^{\varepsilon k+r_{i}^{\varepsilon}} \frac{\partial u}{\partial x_{i}}\left(s, x_{i}^{\prime}\right) d s\right|^{2}\right) d x_{i}^{\prime} \leq 2\left(r_{i}^{\varepsilon}\right)^{2} \int_{I_{r_{i}^{\varepsilon}}^{k} \times \Omega^{\prime}}\left|\frac{\partial u}{\partial x_{i}}\right|^{2},
\end{aligned}
$$

which is sufficient to prove (26).

Proposition 3.3 has a straightforward consequence:

Proposition 3.4. For any $u \in L^{2}\left(0, T ; H^{1}(\Omega)\right)$ and $i \in\{1,2,3\}$, we have

$$
\begin{aligned}
\int_{0}^{T} f_{D_{\varepsilon}^{i}}\left|G_{\varepsilon}^{i}(u)-u\right|^{2} & \leq \varepsilon r_{\varepsilon}^{i}\left|\frac{\partial u}{\partial x_{i}}\right|_{L^{2}\left(\left(D_{\varepsilon}^{i}\right)^{T}\right)}^{2} \\
\left|G_{\varepsilon}^{i}(u)-u\right|_{L^{2}\left(\Omega^{T}\right)} & \leq \varepsilon\left|\frac{\partial u}{\partial x_{i}}\right|_{L^{2}\left(\Omega^{T}\right)}
\end{aligned}
$$

Definition 3.5. For every $\varphi \in L^{2}\left(0, T ; C(\bar{\Omega}) \cap H_{0}^{1}(\Omega)\right)$, define

$$
M_{r_{i}^{\varepsilon}}(\varphi)=\sum_{k \in \mathbf{Z}_{\varepsilon}}\left(f_{I_{\varepsilon}^{k}} \varphi\left(s, x_{i}^{\prime}\right) d s\right) 1_{I_{r_{\varepsilon}}^{k}}\left(x_{i}\right) \in L^{2}\left(\left(D_{\varepsilon}^{i}\right)^{T}\right)
$$

Proposition 3.6. For every $\varphi \in L^{2}\left(0, T ; C(\bar{\Omega}) \cap H_{0}^{1}(\Omega)\right)$, there holds

$$
\lim _{\varepsilon \rightarrow 0} \int_{0}^{T} f_{D_{\varepsilon}^{i}}\left|\varphi-M_{r_{i}^{\varepsilon}}(\varphi)\right|^{2}=0 .
$$

Proof. Let $\varphi \in L^{2}\left(0, T ; C(\bar{\Omega}) \cap H_{0}^{1}(\Omega)\right)$ and let $\delta>0$. The uniform continuity of $\varphi$ yields the existence of some $\varepsilon_{0}>0$ such that for almost all $t \in[0, T]$ we have:

$$
\int_{I_{r_{\varepsilon}}^{k}}\left|\varphi\left(t, x_{i}, x_{i}^{\prime}\right)-f_{I_{\varepsilon}^{k}} \varphi\left(t, s, x_{i}^{\prime}\right) d s\right|^{2}<\delta, \quad \forall x_{i}^{\prime} \in \Omega^{\prime}, \quad \forall k \in \mathbf{Z}_{\varepsilon}, \quad \forall \varepsilon \in\left(0, \varepsilon_{0}\right) .
$$

There results

$$
f_{D_{\varepsilon}^{i}}\left|\varphi-M_{r_{i}^{\varepsilon}}(\varphi)\right|=\frac{1}{\left|D_{\varepsilon}^{i}\right|} \sum_{k \in \mathbf{Z}_{\varepsilon}} \int_{I_{r_{\varepsilon}}^{k} \times \Omega^{\prime}}\left|\varphi\left(t, x_{i}, x_{i}^{\prime}\right)-f_{I_{\varepsilon}^{k}} \varphi\left(t, s, x_{i}^{\prime}\right) d s\right| \leq \delta, \forall \varepsilon \in\left(0, \varepsilon_{0}\right) .
$$

Proposition 3.7. There holds

$$
f_{D_{\varepsilon}^{i}} G_{\varepsilon}^{i}\left(u^{\varepsilon}\right) M_{r_{i}^{\varepsilon}}(\varphi)=f_{\Omega} G_{\varepsilon}^{i}\left(u^{\varepsilon}\right) \varphi, \quad \forall \varphi \in L^{2}\left(0, T ; C(\bar{\Omega}) \cap H_{0}^{1}(\Omega)\right) .
$$

Proof. Let $\varphi \in L^{2}\left(0, T ; C(\bar{\Omega}) \cap H_{0}^{1}(\Omega)\right)$. Uniformly with respect to $t \in[0, T]$, we have:

$$
\begin{aligned}
& f_{D_{\varepsilon}^{i}} G_{\varepsilon}^{i}\left(u^{\varepsilon}\right) M_{r_{i}^{\varepsilon}}(\varphi)=\frac{1}{\left|D_{\varepsilon}^{i}\right|} \sum_{k \in \mathbf{Z}_{\varepsilon}} \int_{I_{r_{\varepsilon}}^{k} \times \Omega^{\prime}} G_{\varepsilon, k}^{i}\left(u^{\varepsilon}\right)\left(x_{i}^{\prime}\right)\left(f_{I_{\varepsilon}^{k}} \varphi\left(s, x_{i}^{\prime}\right) d s\right) d x= \\
& =\frac{1}{\varepsilon \operatorname{card} \mathbf{Z}_{\varepsilon}\left|\Omega^{\prime}\right|} \sum_{k \in \mathbf{Z}_{\varepsilon}} \int_{I_{\varepsilon}^{k} \times \Omega^{\prime}} G_{\varepsilon, k}^{i}\left(u^{\varepsilon}\right)\left(x_{i}^{\prime}\right) \varphi\left(s, x_{i}^{\prime}\right) d x_{i}^{\prime} d s=\frac{1}{L\left|\Omega^{\prime}\right|} \int_{\Omega} G_{\varepsilon}^{i}\left(u^{\varepsilon}\right) \varphi .
\end{aligned}
$$


Corresponding to the inequalities that have already been proved in $H_{0}^{1}(\Omega)$, (see [2], [5], [8] and [9]), we have:

Proposition 3.8. There exists $C>0$, independent of $\varepsilon$, such that, for any $u \in$ $L^{2}\left(0, T ; H_{0}^{1}(\Omega)\right)$ and any $i, j \in\{1,2,3\}$, we have

$$
\begin{gathered}
\int_{0}^{T} f_{D_{\varepsilon}^{i}}|u|^{2} \leq C|\nabla u|_{L^{2}\left(\Omega^{T}\right)}^{2} \\
\int_{0}^{T} f_{D_{\varepsilon}^{i j}}|u|^{2} \leq C \max \left(1, \varepsilon^{2} \ln \frac{1}{r_{i}^{\varepsilon}}, \varepsilon^{2} \ln \frac{1}{r_{j}^{\varepsilon}}\right)|\nabla u|_{L^{2}\left(\Omega^{T}\right)}^{2} \\
\int_{0}^{T} f_{D_{\varepsilon}^{0}}|u|^{2} \leq C \max _{k=1,2,3}\left(1, \frac{\varepsilon^{3}}{r_{k}^{\varepsilon}}\right)|\nabla u|_{L^{2}\left(\Omega^{T}\right)}^{2}
\end{gathered}
$$

where

$$
D_{\varepsilon}^{i j}=D_{\varepsilon}^{i} \cap D_{\varepsilon}^{j}, \quad D_{\varepsilon}^{0}=\cap_{i=1}^{3} D_{\varepsilon}^{i} .
$$

One of the main properties of this box-type microstructure is the following:

Theorem 3.9. There exists $C>0$, independent of $\varepsilon$, such that

$$
f_{D_{\varepsilon}}|u|^{2} \leq C|\nabla u|_{L^{2}\left(\Omega^{T}\right)}^{2}, \quad \forall u \in L^{2}\left(0, T ; H_{0}^{1}(\Omega)\right) .
$$

Proof. Let us notice that

$$
f_{D_{\varepsilon}}|u|^{2} \leq \sum_{i=1}^{3} f_{D_{\varepsilon}^{i}}|u|^{2} \leq 2 \sum_{i=1}^{3} f_{D_{\varepsilon}^{i}}\left(\left|G_{\varepsilon}^{i}(u)-u\right|^{2}+\left|G_{\varepsilon}^{i}(u)\right|^{2}\right) .
$$

Using (27) and (24), it yields

$$
f_{D_{\varepsilon}}|u|^{2} \leq 2\left(\max _{i=1,2,3} r_{\varepsilon}^{i}\right)|\nabla u|_{\Omega}^{2}+4 \sum_{i=1}^{2}\left(\left|G_{\varepsilon}^{i}(u)-u\right|_{\Omega}^{2}+|u|_{\Omega}^{2}\right)
$$

and the proof is completed by (28) and the Poincaré-Friedrichs inequality in $\Omega$.

As the vanishing highly conductive walls have thicknesses of size $r_{i}^{\varepsilon}, 0<r_{i}^{\varepsilon}<<\varepsilon$, we find the asymptotic behaviour of $u^{\varepsilon}$ by applying a control-zone method, that is an energetic method using test-functions associated to a set of much thicker layers which includes the walls but which is still a vanishing set.

More precisely, for any $i \in\{1,2,3\}$, let $\left(R_{i}^{\varepsilon}\right)_{\varepsilon}$ such that $r_{i}^{\varepsilon}<<R_{i}^{\varepsilon}<<\varepsilon$; we define the control-zone of the present problem by

$$
\mathcal{C}_{\varepsilon}=\cup_{i=1}^{3} \mathcal{C}_{\varepsilon}^{i}, \quad \mathcal{C}_{\varepsilon}^{i}=\cup_{k \in \mathbf{Z}_{\varepsilon}} \mathcal{C}_{\varepsilon, k}^{i},
$$

where for any $k \in \mathbf{Z}_{\varepsilon}$ we have

$$
\mathcal{C}_{\varepsilon, k}^{i}=\left\{x=\left(x_{i}, x_{2}, x_{3}\right) \in \Omega, \quad\left|x_{i}-\varepsilon k\right|<R_{i}^{\varepsilon}\right\} .
$$

We associate to our control-zone the following functions $w_{\varepsilon}^{i} \in W^{1, \infty}(\Omega)$ :

$$
w_{\varepsilon}^{i}(x)= \begin{cases}1-\frac{r_{i}^{\varepsilon}}{R_{i}^{\varepsilon}} & \text { if } \quad x=\left(x_{i}, x_{2}, x_{3}\right) \in D_{\varepsilon}^{i} \\ 1-\frac{\left|x_{i}-\varepsilon k\right|}{R_{i}^{\varepsilon}} & \text { if } \quad x \in\left(\mathcal{C}_{\varepsilon, k}^{i} \backslash D_{\varepsilon, k}^{i}\right) \text { for some } k \in \mathbf{Z}_{\varepsilon} \\ 0 & \text { if } \quad x \in \Omega \backslash \mathcal{C}_{\varepsilon}^{i} .\end{cases}
$$


Also, to any $\varphi \in \mathcal{D}(\Omega)$ we associate a step approximation function $\varphi_{i}^{\varepsilon} \in L^{\infty}(\Omega)$ defined with respect to the control-zone:

$$
\varphi_{i}^{\varepsilon}\left(x_{i}, x_{2}, x_{3}\right)=\left.\sum_{k \in \mathbf{Z}_{\varepsilon}} \varphi\right|_{x_{i}=\varepsilon k}\left(x_{i}^{\prime}\right) 1_{I_{R_{i}^{\varepsilon}}^{k}}\left(x_{i}\right),
$$

where $I_{R_{i}^{\varepsilon}}^{k}:=\varepsilon k+2 R_{i}^{\varepsilon} I$.

The basic properties of these functions can be easily obtained:

$$
\begin{gathered}
\left|\nabla w_{\varepsilon}^{i}\right|_{\mathcal{C}_{\varepsilon}} \leq\left(\frac{2|\Omega|}{\varepsilon R_{i}^{\varepsilon}}\right)^{1 / 2} \\
\left|\varphi-\varphi_{i}^{\varepsilon}\right|_{L^{\infty}\left(\mathcal{C}_{\varepsilon}^{i}\right)} \leq R_{i}^{\varepsilon}|\nabla \varphi|_{L^{\infty}(\Omega)} . \\
\left|\nabla \varphi_{i}^{\varepsilon}\right|_{\mathcal{C}_{\varepsilon}^{i}} \leq\left(2|\Omega| \frac{R_{i}^{\varepsilon}}{\varepsilon}\right)^{1 / 2}|\nabla \varphi|_{L^{\infty}(\Omega)} .
\end{gathered}
$$

\section{The homogenization procedure}

For any $i \in\{1,2,3\}$ we assume that there exist

$$
m_{i}=\lim _{\varepsilon \rightarrow 0} \frac{\left|D_{\varepsilon}^{i}\right|}{\left|D_{\varepsilon}\right|} \in[0,1] .
$$

Obviously, we have

$$
m_{1}+m_{2}+m_{3}=1 \text {. }
$$

This situation covers all the following three types of structures: honeycomb, gridwork and layered (see [12]).

We can present now the preliminary convergence results:

Proposition 4.1. There exists $u \in L^{\infty}\left(0, T ; L^{2}(\Omega)\right) \cap L^{2}\left(0, T ; H_{0}^{1}(\Omega)\right)$ such that, on some subsequence,

$$
\begin{gathered}
u^{\varepsilon} \stackrel{\star}{\rightarrow} u \quad \text { in } \quad L^{\infty}\left(0, T ; L^{2}(\Omega)\right) \\
u^{\varepsilon} \rightarrow u \quad \text { in } \quad L^{2}\left(0, T ; H_{0}^{1}(\Omega)\right) \\
G_{\varepsilon}^{i}\left(u^{\varepsilon}\right) \rightarrow u \quad \text { in } \quad L^{2}\left(\Omega^{T}\right), \quad \forall i \in\{1,2,3\} \\
\int_{0}^{T} f_{D_{\varepsilon}} u^{\varepsilon} v \rightarrow \int_{0}^{T} f_{\Omega} u v, \quad \forall v \in L^{2}\left(0, T ; H_{0}^{1}(\Omega)\right)
\end{gathered}
$$

Moreover, for any $i \in\{1,2,3\}$ we have:

$\int_{0}^{T} f_{D_{\varepsilon}^{i}} \frac{\partial u^{\varepsilon}}{\partial x_{j}} v \rightarrow \int_{0}^{T} f_{\Omega} \frac{\partial u}{\partial x_{j}} v, \quad \forall v \in L^{2}\left(0, T ; H_{0}^{1}(\Omega)\right), \quad \forall j \in\{1,2,3\}, \quad j \neq i$.

Proof. The weak convergences (44)-(45) follow from (19). As $H_{0}^{1}(\Omega)$ is compactly embedded in $L^{2}(\Omega)$, the strong convergence (46) is obtained by using (28) and (45). In order to prove (47) we remark that

$\int_{0}^{T} f_{D_{\varepsilon}} u^{\varepsilon} v=\frac{\left|D_{\varepsilon}^{0}\right|}{\left|D_{\varepsilon}\right|} \int_{0}^{T} f_{D_{\varepsilon}^{0}} u^{\varepsilon} v-\sum_{1 \leq i<j \leq 3} \frac{\left|D_{\varepsilon}^{i j}\right|}{\left|D_{\varepsilon}\right|} \int_{0}^{T} f_{D_{\varepsilon}^{i j}} u^{\varepsilon} v+\sum_{i=1}^{3} \frac{\left|D_{\varepsilon}^{i}\right|}{\left|D_{\varepsilon}\right|} \int_{0}^{T} f_{D_{\varepsilon}^{i}} u^{\varepsilon} v$ 
Using (20), (32) and (33), we prove that the first two terms of (49) are converging to zero, as follows:

$$
\begin{gathered}
\left|\frac{\left|D_{\varepsilon}^{0}\right|}{\left|D_{\varepsilon}\right|} \int_{0}^{T} f_{D_{\varepsilon}^{0}} u_{\varepsilon} v\right| \leq C \frac{\left|D_{\varepsilon}^{0}\right|}{\left|D_{\varepsilon}\right|} \max \left(1, \frac{\varepsilon^{3}}{r_{\varepsilon}}\right)|\nabla v|_{\Omega} \rightarrow 0, \\
\left|\frac{\left|D_{\varepsilon}^{i j}\right|}{\left|D_{\varepsilon}\right|} \int_{0}^{T} f_{D_{\varepsilon}^{i j}} u_{\varepsilon} v\right| \leq C \frac{\left|D_{\varepsilon}^{i j}\right|}{\left|D_{\varepsilon}\right|} \max \left(1, \varepsilon^{2} \ln \frac{1}{r_{\varepsilon}^{i}}, \varepsilon^{2} \ln \frac{1}{r_{\varepsilon}^{j}}\right)|\nabla v|_{\Omega} \rightarrow 0 .
\end{gathered}
$$

It follows that

$$
\lim _{\varepsilon \rightarrow 0} \int_{0}^{T} f_{D_{\varepsilon}} u^{\varepsilon} v=\sum_{i=1}^{3} m_{i} \lim _{\varepsilon \rightarrow 0} \int_{0}^{T} f_{D_{\varepsilon}^{i}} u^{\varepsilon} v
$$

Let $\varphi \in L^{2}(0, T ; \mathcal{D}(\Omega))$. We have

$$
\int_{0}^{T} f_{D_{\varepsilon}^{i}} u^{\varepsilon} \varphi=\int_{0}^{T} f_{D_{\varepsilon}^{i}}\left(u^{\varepsilon}-G_{\varepsilon}^{i}\left(u^{\varepsilon}\right)\right) \varphi+\int_{0}^{T} f_{D_{\varepsilon}^{i}} G_{\varepsilon}^{i}\left(u^{\varepsilon}\right)\left(\varphi-M_{r_{i}^{\varepsilon}}(\varphi)\right)+\int_{0}^{T} f_{D_{\varepsilon}^{i}} G_{\varepsilon}^{i}\left(u^{\varepsilon}\right) M_{r_{i}^{\varepsilon}}(\varphi) \text {. }
$$

Taking into account (26) and (29), we have:

$$
\begin{aligned}
& \left|\int_{0}^{T} f_{D_{\varepsilon}^{i}}\left(u^{\varepsilon}-G_{\varepsilon}^{i}\left(u^{\varepsilon}\right)\right) \varphi\right| \leq C r_{i}^{\varepsilon}\left(\int_{0}^{T} f_{D_{\varepsilon}^{i}}\left|\frac{\partial u_{\varepsilon}}{\partial x_{i}}\right|^{2}\right)^{1 / 2}|\varphi|_{L^{2}\left(0, T ; L^{\infty}(\Omega)\right)} \leq C r_{i}^{\varepsilon} \rightarrow 0 \\
& \left|\int_{0}^{T} f_{D_{\varepsilon}^{i}} G_{\varepsilon}^{i}\left(u^{\varepsilon}\right)\left(\varphi-M_{r_{i}^{\varepsilon}}(\varphi)\right)\right| \leq\left|G_{\varepsilon}^{i}\left(u^{\varepsilon}\right)\right|_{L^{2}\left(\Omega^{T}\right)}\left(\int_{0}^{T} f_{D_{\varepsilon}^{i}}\left|\varphi-M_{r_{i}^{\varepsilon}}(\varphi)\right|^{2}\right)^{1 / 2} \rightarrow 0 .
\end{aligned}
$$

Then, using (30), we get

$$
\lim _{\varepsilon \rightarrow 0} \int_{0}^{T} f_{D_{\varepsilon}^{i}} u^{\varepsilon} \varphi=\lim _{\varepsilon \rightarrow 0} \int_{0}^{T} f_{\Omega} G_{\varepsilon}^{i}\left(u^{\varepsilon}\right) \varphi=\int_{0}^{T} f_{\Omega} u \varphi .
$$

In order to conclude with $(47)$, we notice that $L^{2}(0, T ; \mathcal{D}(\Omega))$ is dense in $L^{2}\left(0, T ; H_{0}^{1}(\Omega)\right)$ and that the following estimate proves the continuity of the mapping $\varphi \mapsto \int_{0}^{T} f_{D_{\varepsilon}^{i}} u^{\varepsilon} \varphi$ :

$$
\left|\int_{0}^{T} f_{D_{\varepsilon}^{i}} u^{\varepsilon} \varphi\right| \leq\left(\int_{0}^{T} f_{D_{\varepsilon}^{i}}\left|u^{\varepsilon}\right|^{2}\right)^{1 / 2}\left(\int_{0}^{T} f_{D_{\varepsilon}^{i}}|\varphi|^{2}\right)^{1 / 2} \leq C|\nabla \varphi|_{L^{2}\left(\Omega^{T}\right)} .
$$

Next, let again $\varphi \in L^{2}(0, T ; \mathcal{D}(\Omega))$ and $i \in\{1,2,3\}$. Denoting by $\nu=\left(\nu_{1}, \nu_{2}, \nu_{3}\right)$ the outward normal to $\partial D_{\varepsilon}$ we obviously have $\varphi \nu_{j}=0$ on $\left(\partial D_{\varepsilon}^{i}\right)^{T}(j \neq i)$ and therefore:

$$
\int_{0}^{T} f_{D_{\varepsilon}^{i}} \frac{\partial u^{\varepsilon}}{\partial x_{j}} \varphi=-\int_{0}^{T} f_{D_{\varepsilon}^{i}} u^{\varepsilon} \frac{\partial \varphi}{\partial x_{j}} .
$$

Using (51), from the previous relation it follows:

$$
\int_{0}^{T} f_{D_{\varepsilon}^{i}} \frac{\partial u^{\varepsilon}}{\partial x_{j}} \varphi \rightarrow-\int_{0}^{T} f_{\Omega} u \frac{\partial \varphi}{\partial x_{j}}=\int_{0}^{T} f_{\Omega} \frac{\partial u}{\partial x_{j}} \varphi .
$$

The proof of (48) is completed by continuity, using (20) and (35).

Now we can present our main result.

Theorem 4.2. Assume that

$$
\begin{gathered}
u_{0}^{\varepsilon} \rightarrow u_{0} \quad \text { in } \quad L^{2}(\Omega) \\
f^{\varepsilon} \rightarrow f \quad \text { in } \quad L^{2}\left(0, T ; H^{-1}(\Omega)\right) .
\end{gathered}
$$


Then, there exists $u \in L^{\infty}\left(0, T ; L^{2}(\Omega)\right) \cap L^{2}\left(0, T ; H_{0}^{1}(\Omega)\right)$ such that

$$
\begin{array}{lll}
u \stackrel{\star}{\rightarrow} \quad \text { in } \quad L^{\infty}\left(0, T ; L^{2}(\Omega)\right) \\
u \rightarrow u \quad \text { in } \quad L^{2}\left(0, T ; H_{0}^{1}(\Omega)\right) .
\end{array}
$$

The limit $u \in L^{2}\left(0, T ; H_{0}^{1}(\Omega)\right)$ is the only solution of the equation

$$
\begin{gathered}
\rho_{a} \frac{\partial u}{\partial t}-\sum_{i, j=1}^{3} \frac{\partial}{\partial x_{i}}\left(a_{i j} \frac{\partial u}{\partial x_{j}}\right)-\sum_{i=1}^{3} \frac{\left(1-m_{i}\right)}{|\Omega|} \frac{\partial}{\partial x_{i}}\left(b_{i} \frac{\partial u}{\partial x_{i}}\right)=f \quad \text { in } \quad L^{2}\left(0, T ; H^{-1}(\Omega)\right) \\
u(0)=u_{0} \quad \text { in } \quad L^{2}(\Omega)
\end{gathered}
$$

Proof. Let $\eta \in \mathcal{D}\left(\left[0, T[), v \in H_{0}^{1}(\Omega)\right.\right.$. We have

$$
\begin{aligned}
& -\int_{0}^{T} \eta^{\prime} \int_{\Omega \backslash D_{\varepsilon}} \rho_{a} u^{\varepsilon} v+\sum_{i, j=1}^{3} \int_{0}^{T} \eta \int_{\Omega \backslash D_{\varepsilon}} a_{i j} \frac{\partial u^{\varepsilon}}{\partial x_{j}} \frac{\partial v}{\partial x_{i}}+\int_{0}^{T} \eta f_{D_{\varepsilon}}\left(\sum_{k=1}^{3} b_{k} \frac{\partial u^{\varepsilon}}{\partial x_{k}} \frac{\partial v}{\partial x_{k}}\right)= \\
& =\int_{0}^{T} \eta^{\prime} \int_{D_{\varepsilon}} \rho_{b} u^{\varepsilon} v+\eta(0) \int_{\Omega \backslash D_{\varepsilon}} \rho_{a} u_{0}^{\varepsilon} v+\eta(0) \int_{D_{\varepsilon}} \rho_{b} u_{0}^{\varepsilon} v+\int_{0}^{T} \eta\left\langle f^{\varepsilon}, v\right\rangle_{H^{-1}, H_{0}^{1}(\Omega)} .
\end{aligned}
$$

Recalling (19) and (20) we find that on some subsequence each of the following terms has a limit, defined by

$$
B_{i}=\lim _{\varepsilon \rightarrow 0} \int_{0}^{T} \eta f_{D_{\varepsilon}^{i}}\left(\sum_{k=1}^{3} b_{k} \frac{\partial u^{\varepsilon}}{\partial x_{k}} \frac{\partial v}{\partial x_{k}}\right), \quad i \in\{1,2,3\} .
$$

Taking the limit of $(58), \varepsilon \rightarrow 0$, we immediately obtain:

$$
\begin{gathered}
-\int_{0}^{T} \eta^{\prime} \int_{\Omega} \rho_{a} u v+\sum_{i, j=1}^{3} \int_{0}^{T} \eta \int_{\Omega} a_{i j} \frac{\partial u}{\partial x_{j}} \frac{\partial v}{\partial x_{i}}+\sum_{i=1}^{3} m_{i} B_{i}= \\
=\eta(0) \int_{\Omega} \rho_{a} u_{0}^{\varepsilon} v+\int_{0}^{T} \eta\langle f, v\rangle_{H^{-1}, H_{0}^{1}(\Omega)} .
\end{gathered}
$$

It remains to identify $B_{i}$.

Let $\varphi \in \mathcal{D}(\Omega)$. For any $i \in\{1,2,3\}$, we set $v=v_{i}^{\varepsilon}:=\left(\varphi-\varphi_{i}^{\varepsilon}\right) w_{i}^{\varepsilon}$ in $(60)$.

Let us remark that $v_{i}^{\varepsilon} \rightarrow 0$ strongly in $H_{0}^{1}(\Omega)$, as a straightforward consequence of the following estimate

$$
\int_{\Omega}\left|\nabla v_{i}^{\varepsilon}\right|^{2} \leq 2 \int_{\mathcal{C}_{\varepsilon}^{i}}\left|\nabla w_{i}^{\varepsilon}\right|^{2}\left|\varphi-\varphi_{i}^{\varepsilon}\right|^{2}+2 \int_{\mathcal{C}_{\varepsilon}^{i}}\left|w_{i}^{\varepsilon}\right|^{2}\left|\nabla\left(\varphi-\varphi_{i}^{\varepsilon}\right)\right|^{2} \leq C\left(\frac{R_{i}^{\varepsilon}}{\varepsilon}\right) .
$$

After substitution of $v=v_{i}^{\varepsilon}$ in (60) and taking advantage of the property that $v_{i}^{\varepsilon} \neq 0$ only in $\mathcal{C}_{\varepsilon}^{i}$, we obtain

$$
m_{i} \lim _{\varepsilon \rightarrow 0} \int_{0}^{T} \eta f_{D_{\varepsilon}^{i}}\left(\sum_{k=1}^{3} b_{k} \frac{\partial u^{\varepsilon}}{\partial x_{k}} \frac{\partial\left(\varphi-\varphi_{i}^{\varepsilon}\right)}{\partial x_{k}}\right)=0
$$

that is,

$$
\begin{gathered}
m_{i} B_{i}=m_{i} \lim _{\varepsilon \rightarrow 0} \sum_{h \in \mathbf{Z}_{\varepsilon}} \varepsilon \int_{0}^{T} \eta f_{D_{\varepsilon, h}^{i}}\left(\sum_{\substack{k=1 \\
k \neq i}}^{3} b_{k} \frac{\partial u^{\varepsilon}}{\partial x_{k}} \frac{\partial \varphi}{\partial x_{k}}\left(\varepsilon h, x_{i}^{\prime}\right)\right)= \\
=m_{i} \lim _{\varepsilon \rightarrow 0} \int_{0}^{T} \eta f_{D_{\varepsilon}^{i}}\left(\sum_{\substack{k=1 \\
k \neq i}}^{3} b_{k} \frac{\partial u^{\varepsilon}}{\partial x_{k}} \frac{\partial \varphi}{\partial x_{k}}\right)=
\end{gathered}
$$


ASYMPTOTIC HEAT EQUATION FOR CROSSING SUPERCONDUCTIVE THIN WALLS 11

$$
=-m_{i} \lim _{\varepsilon \rightarrow 0} \int_{0}^{T} \eta f_{D_{\varepsilon}^{i}}\left(\sum_{\substack{k=1 \\ k \neq i}}^{3} u^{\varepsilon} \frac{\partial}{\partial x_{k}}\left(b_{k} \frac{\partial \varphi}{\partial x_{k}}\right)\right) .
$$

The regularity property $b_{k} \in C^{2}(\bar{\Omega})$ for every $k \in\{1,2,3\}$ implies that

$$
\frac{\partial}{\partial x_{k}}\left(b_{k} \frac{\partial \varphi}{\partial x_{k}}\right) \in H_{0}^{1}(\Omega)
$$

and that integration by parts holds true, thus yielding

$$
m_{i} B_{i}=-m_{i} \int_{0}^{T} \eta f_{\Omega} \sum_{\substack{k=1 \\ k \neq i}}^{3} u \frac{\partial}{\partial x_{k}}\left(b_{k} \frac{\partial \varphi}{\partial x_{k}}\right)=m_{i} \int_{0}^{T} \eta f_{\Omega} \sum_{\substack{k=1 \\ k \neq i}}^{3} b_{k} \frac{\partial u}{\partial x_{k}} \frac{\partial \varphi}{\partial x_{k}} .
$$

Summing over $i \in\{1,2,3\}$, we obtain:

$$
\begin{gathered}
\sum_{i=1}^{3} m_{i} B_{i}=\sum_{i=1}^{3} m_{i} \int_{0}^{T} \eta f_{\Omega} \sum_{\substack{k=1 \\
k \neq i}}^{3} b_{k} \frac{\partial u}{\partial x_{k}} \frac{\partial \varphi}{\partial x_{k}}=\int_{0}^{T} \eta f_{\Omega} \sum_{k=1}^{3} b_{k} \frac{\partial u}{\partial x_{k}} \frac{\partial \varphi}{\partial x_{k}}- \\
-\sum_{i=1}^{3} m_{i} \int_{0}^{T} \eta f_{\Omega} b_{i} \frac{\partial u}{\partial x_{i}} \frac{\partial \varphi}{\partial x_{i}}=\sum_{i=1}^{3}\left(1-m_{i}\right) \int_{0}^{T} \eta f_{\Omega} b_{i} \frac{\partial u}{\partial x_{i}} \frac{\partial \varphi}{\partial x_{i}}
\end{gathered}
$$

Substituting (66) into (60) and using the density of $\mathcal{D}(\Omega)$ in $H_{0}^{1}(\Omega)$, we obtain by continuity:

$$
\begin{aligned}
-\int_{0}^{T} \eta^{\prime} \int_{\Omega \backslash D_{\varepsilon}} \rho_{a} u v+ & \sum_{i, j=1}^{3} \int_{0}^{T} \eta \int_{\Omega} a_{i j} \frac{\partial u}{\partial x_{j}} \frac{\partial v}{\partial x_{i}}+\sum_{i=1}^{3}\left(1-m_{i}\right) \int_{0}^{T} \eta f_{\Omega} b_{i} \frac{\partial u}{\partial x_{i}} \frac{\partial v}{\partial x_{i}}= \\
& =\eta(0) \int_{\Omega} \rho_{a} u_{0}^{\varepsilon} v+\int_{0}^{T} \eta\langle f, v\rangle_{H^{-1}, H_{0}^{1}(\Omega)} .
\end{aligned}
$$

which obviously completes the proof.

Remark 4.3. The reticulated domain occupied by all the intersections of the layers has no distinct influence upon the asymptotic distribution of the temperature. For $i<j$ and $h \in\{i, j\}$, this follows from

$$
\begin{gathered}
\left|\frac{1}{\left|D_{\varepsilon}\right|} \int_{D_{\varepsilon}^{i j}} \sum_{k=1}^{3} b_{k} \frac{\partial u_{\varepsilon}}{\partial x_{k}} \frac{\partial \varphi_{h}^{\varepsilon}}{\partial x_{k}}\right| \leq C|\nabla \varphi|_{L^{\infty}(\Omega)}\left(\frac{1}{\left|D_{\varepsilon}\right|} \int_{D_{\varepsilon}^{i j}}\left|\nabla u_{\varepsilon}\right|^{2}\right)^{\frac{1}{2}}\left(\frac{\left|D_{\varepsilon}^{i j}\right|}{\left|D_{\varepsilon}\right|}\right)^{\frac{1}{2}} \leq \\
\leq C|\nabla \varphi|_{L^{\infty}(\Omega)}\left(\frac{r_{\varepsilon}}{\varepsilon}\right)^{\frac{1}{2}} \rightarrow 0,
\end{gathered}
$$

where we have used (32).

Remark 4.4. Our main result works for any $m_{i} \geq 0$, that is, it covers the three main structures of this type:

- the honeycomb (box-type) structures, which correspond to the case when $m_{i}>0, \forall i \in\{1,2,3\}$,

- the gridworks, which correspond to the case when only one of the $m_{i}$ 's is zero,

- the layered structures which correspond to the case when two of the $m_{i}$ 's are zero. 
Acknowledgements. This work was done during the visit of Dan Polişevschi at the I.R.M.A.R.'s Department of Mechanics (University of Rennes 1) whose support is gratefully acknowledged.

\section{REFERENCES}

[1] N.S. Bakhvalov, G.P. Panasenko, Homogenization: averaging processes in periodic media, Math. Appl. (Sov. Series), 36, Kluwer Acad. Pub. Group, Dordrecht, 1989.

[2] M. Bellieud, G. Bouchitté, Homogenization of elliptic problems in a fiber reinforced structure. Non local effects, Ann. Scuola Norm. Sup. Pis Cl. Sci.(4), 26(3), 1998, 407-436.

[3] F. Bentalha, I. Gruais, D. Polisevski, Homogenization of a conductive suspension in a Stokes-Boussinesq flow, Applicable Analysis, 85(6-7), 2006, 811-830.

[4] F. Bentalha, I. Gruais, D. Polisevssi, Diffusion process in a rarefied binary structure, Revue Roumaine de Mathématiques Pures et Appliquées, 52(1), 2007, 9-34.

[5] F. Bentalha, I. Gruais, D. Polisevski, Diffusion in a highly rarefied binary structure of general periodic shape, Applicable Analysis, 87(6), 2008, 635-655.

[6] G. Bouchitté, C. Picard, Singular perturbations and homogenization in stratified media, Applicable Analysis, 61(3-4), 1996, 307-341.

[7] I. Gruais, D. Polisevski, Homogenizing a critical binary structure of finite diffusivities, Asymptotic Analysis, 55(1), 2007, 85-102.

[8] I. Gruais, D. Polisevski, Periodic structures separated by highly conductive thin layers, Prépublication IRMAR, 08-28, 2008, 1-21.

[9] I. Gruais, D. Polisevski, Homogenizing media containing a highly conductive honeycomb substructure, Asymptotic Analysis, 67(1-2), 2010, 33-43.

[10] B. Héron, J. Mossino, C. Picard, Homogenization of some quasilinear problems for stratified media with low and high conductivities, Differential Integral Equations, 7(1), 1994, $157-178$.

[11] F. Murat, L. Tartar, Calcul des variations et homogénéisation, in " Homogenization methods: theory and applications in physics", Collect. Dir. Études Rech. Élec. France, 57, Eyrolles, Paris, 1985, 319-370.

[12] D. Cioranescu, J. Saint-Jean-Paulin, Homogenization of reticulated structures, SpringerVerlag, New-York, 1999.

[13] V.V. Zhikov, S.E. Pastukhova, Derivation of the limit equations of elasticity theory of thin sets, Journal of Mathematical Sciences, 135(1), 2006, 2637-2665.

* Université de Rennes 1, I.R.M.A.R, Campus de Beaulieu, 35042 Rennes Cedex (France)

** Institutul de Matematica "S. Stoilow", Academia Romana, P.O. Box 1-764, Bucureşti (Romania). 